\title{
Phylum-specific environmental DNA analysis reveals remarkably high global biodiversity of Cercozoa (Protozoa)
}

\author{
Correspondence \\ David Bass \\ david.bass@zoo.ox.ac.uk
}

\author{
David Bass and Thomas Cavalier-Smith \\ Department of Zoology, University of Oxford, South Parks Road, Oxford OX1 3PS, UK
}

\section{INTRODUCTION}

Cercozoa include many of the most abundant and therefore ecologically significant soil, freshwater and marine protozoa. When the phylum was first described (CavalierSmith, 1996/7, 1998a, b), it was the first major eukaryotic group to be recognized primarily as a result of molecular phylogenetic analyses rather than by morphological characteristics. At that time, three subphyla were recognized: Phytomyxa, equivalent to the current Phytomyxea, and Monadofilosa and Reticulofilosa, both now treated as superclasses (Cavalier-Smith \& Chao, 2003). All included taxa had previously been of uncertain affinity. By the time of the most recent and thorough description of Cercozoa (Cavalier-Smith \& Chao, 2003), the phylum was considerably expanded by the addition of the parasitic Ascetosporea, Gromiidea (Burki et al., 2002), several filose testate amoebae (Wylezich et al., 2002), Cryothecomonas (Kühn et al., 2000), Proleptomonas (Vickerman et al., 2002),

Published online ahead of print on 9 July 2004 as DOI 10.1099/ ijs.0.63229-0.

Abbreviations: GTR, general time-reversible; ML, maximum-likelihood; $\mathrm{TBR}$, tree bisection/reconnection.

The GenBank/EMBL/DDBJ accession numbers for the 18S rRNA gene environmental and cultured sequences reported in this study are AY620252-AY620366.
Massisteria (Atkins et al., 2000), Gymnophrys and Lecythium (Nikolaev et al., 2003) and Allantion, Allas, Bodomorpha and Spongomonas (Cavalier-Smith, 2000), as well as several strains not identified to the genus level (Cavalier-Smith \& Chao, 2003), mainly as a result of $18 \mathrm{~S}$ rRNA gene sequences derived from culture-based surveys of a wide range of putative cercozoans. The resulting cercozoan subgroups each show distinct morphological characteristics, but no simple morphological character unites the phylum as a whole.

The past few years have seen an increasing number of environmental $18 \mathrm{~S}$ rRNA gene library surveys of microbial eukaryote diversity in a wide range of environments (e.g. Moon-van der Staay et al., 2001; López-García et al., 2001; reviewed by Moreira \& López-García, 2002). Almost without exception, such surveys have unveiled an unexpectedly large diversity of hitherto unknown eukaryote lineages. However, there has been no intensive study of a single protozoan phylum, using phylum-specific primers to exclude all other eukaryotes from the analysis.

Therefore, we made 41 cercozoan-specific 18S rRNA environmental libraries from a range of habitats on two continents (see Table 1) for several reasons: (i) to gauge the magnitude of total cercozoan diversity, (ii) to detect novel cercozoan lineages in order to facilitate their description 
Table 1. Provenance of samples from which $4118 \mathrm{~S}$ rRNA libraries were created

Each entry in the third column represents a separate library.

\begin{tabular}{|c|c|c|}
\hline Site & Sample details & $\operatorname{Code}(s)$ on trees \\
\hline \multicolumn{3}{|l|}{ British Columbia, Canada } \\
\hline \multirow[t]{4}{*}{ Wreck Beach, Vancouver } & Low tide sand/mud & $9-1 . x, 9-2 . x$ \\
\hline & Mud from near high-tide mark & $9-3 . x, 9-4 \cdot x$ \\
\hline & Woodland stream in woods behind Wreck Beach (freshwater) & $13-1 . x, 2 . x$ \\
\hline & Woodland soil from behind Wreck Beach & $9-5 . x, 9-6 . x, 13-3 . x, 13-4 . x$ \\
\hline Vancouver Island, west coast & Low-tide sand, Long Beach, Tofino & $\mathrm{A} x, \mathrm{D} x$ \\
\hline \multicolumn{3}{|l|}{ Bermuda } \\
\hline Spittal Pond & Freshwater pond sediment & $\mathrm{B} x, 12-1 . x, 12-2 . x$ \\
\hline \multicolumn{3}{|l|}{ UK } \\
\hline \multirow[t]{3}{*}{ Portsmouth area coast } & Muddy sand, Southsea Beach & $7-1 . x, 7-2 . x$ \\
\hline & Muddy sand, Bosham harbour & $7-3 . x, 7-4 . x$ \\
\hline & Muddy sand, Portsmouth waterfront & $7-5 . x, 7-6 \cdot x$ \\
\hline Treath Beach, west Wales & Filtered surf water & $C x, 12-3 . x, 12-4 . x$ \\
\hline \multirow[t]{7}{*}{ Priest Pot (freshwater pond), Cumbria } & $0.5 \mathrm{~m}$ depth water column, filtered & $\mathrm{F} x$ \\
\hline & Leafy sediment & PP1.x, PP2.x \\
\hline & $0.5 \mathrm{~m}$ depth sediment & $6-1 . x, 6-2 \cdot x$ \\
\hline & $3 \cdot 1 \mathrm{~m}$ depth sediment & $6-3 . x, 6-4 \cdot x$ \\
\hline & Sphagnum moss from edge of pond & $4-1 . x, 4-2 \cdot x$ \\
\hline & Wet soil from edge of pond & $4-3 . x, 4-4 \cdot x$ \\
\hline & Scraping from submerged lily stem & $8-1 . x, 8-2 . x, 10-3 . x$ \\
\hline \multicolumn{3}{|l|}{ Spain } \\
\hline Valencia & Garden soil, El Vedat de Torrente & $4-5 . x, 4-6 . x, 10-4 . x$ \\
\hline
\end{tabular}

in the future, and to improve the resolution of the cercozoan tree, and (iii) to describe the distribution of lineages in terms of habitat and geography. For example, do cercozoan lineages show evidence of biogeographical structuring with respect to the evolutionary rate of $18 \mathrm{~S}$ rRNA? This question is important in relation to the recent interest in the extent and rates of global dispersal of microbial eukaryotes (summarized in Finlay, 2002).

Because cercozoan phylogeny is based largely on 18S rRNA gene sequences, the branching order of the major clades remains unclear due to lack of resolution of the relatively rapid basal radiation and to biases and inadequacies inherent in analyses using this gene (Embley \& Hirt, 1998; Stiller \& Hall, 1999; Philippe \& Germot, 2000; CavalierSmith, 2004). However, the sampling of cercozoan $18 \mathrm{~S}$ rRNA gene sequences far exceeds that of any other gene and, as has been shown by Horner \& Embley (2001) and Richards et al. (2003), optimal taxon sampling can be one of the most crucial factors in producing robust phylogenies. Here, by combining new sequences from environmental libraries and pure cultures, and an existing database, we present a phylogenetic analysis using the largest taxon sample for $18 \mathrm{~S}$ rRNA to date for Cercozoa. Other phylogenies based on protein-coding genes and a unique polyubiquitin insertion indicate that the closest relatives of Cercozoa are Foraminifera (Archibald et al., 2003; Longet et al., 2003; Burki et al., 2002; Cavalier-Smith \& Chao, 2003; Keeling, 2001).

\section{METHODS}

Samples were collected in sterile plastic tubes. As soon as possible after collection, DNA was extracted with the UltraClean soil DNA isolation kit (Mo Bio Laboratories), using $0 \cdot 5-0 \cdot 75 \mathrm{~g}$ soil/sediment or $25-40 \mathrm{ml}$ water (for the freshwater and marine water column samples) filtered through a glass-fibre filter (Whatman GF/F), which was cut into small pieces before the first (homogenization) step. Partial 18S rRNA gene sequences were amplified by PCR with primer 25F (5'-CATATGCTTGTCTCAAAGATTAAGCCA-3') and the cercozoan-specific primer 1256R (5'-GCACCACCACCCAYAGAATCAAGAAAGAWC- $3^{\prime}$ ) under the following cycling conditions: 5 min at $95^{\circ} \mathrm{C}, 30$ cycles of $32 \mathrm{~s}$ at $95^{\circ} \mathrm{C}, 36 \mathrm{~s}$ at $70^{\circ} \mathrm{C}$ and $210 \mathrm{~s}$ at $72^{\circ} \mathrm{C}$ and then $8 \mathrm{~min}$ at $72^{\circ} \mathrm{C}$. Typically, these primers amplify a segment of about $1.26 \mathrm{~kb}$. rRNA gene clone libraries were constructed using the TOPO TA cloning kit (Invitrogen). White or light-blue colonies were picked and grown in $2 \mathrm{ml} \mathrm{LB}$ medium plus $2 \mu \mathrm{l} 50 \mathrm{mg}$ ampicillin $\mathrm{ml}^{-1}$ overnight. The plasmid DNA was extracted and purified using the QIAquick PCR purification kit (Qiagen). The purified products were run on a gel with known negative transformants; positive transformants were sequenced using dye terminators and separated on an automated ABI-377 sequencer. The sequencing primers used were M13 forward and M13 reverse from the TOPO TA kit and the internal primer Pre3NDf $\left(5^{\prime}\right.$ CAGCAGGCGCGCAAATTACC-3').

The cloned sequences were edited in the trace editor TED and then aligned by eye with the 91 named (cultured) cercozoan sequences in our database using the Genetic Data Environment software. BioNJ trees were constructed to eliminate non-cercozoan sequences (3\% of all cloned sequences); separate trees were constructed using the first and last 500 positions in order to identify and eliminate putative chimaeric sequences (see Results and Discussion) before further 
phylogenetic analysis was carried out. We have found this to be an effective way of detecting chimaeras. The sequences included in this study passed this test, with the two trees giving essentially the same tree topology. Some putative chimaeric sequences were eliminated from the study after screening with RDP version 1.07 (Martin \& Rybicki, 2000). A subset of taxa was run through CHIMERA_CHECK 2.7 (http:// rdp.cme.msu.edu/cgis/chimera.cgi?su=SSU), but interpretation of the results was difficult due to the low representation of Cercozoa in the databases. Gaps and ambiguously aligned positions were also removed, leaving an alignment of 1164 positions.

ModelTest version 3.06 (Posada \& Crandall, 1998) selected the general time-reversible (GTR) model with gamma correction for intersite rate variation $(\Gamma)$ and allowance for invariant sites (i) for both datasets [189 taxa for distance trees, 76 taxa for maximum-likelihood (ML) trees]. These parameters were used for distance trees constructed using PAUP 4.0b10 (Swofford, 1999). Heuristic searches (500 replicates with a 20 min limit per replicate; random addition) using both minimum evolution and least squares (power 2) were made [with tree bisection/ reconnection (TBR) branch swapping]. The tree was rooted between Filosa and Endomyxa as shown by ML trees with close outgroups in Cavalier-Smith \& Chao (2003). ML trees were also calculated with empirical base frequencies $(\mathrm{GTR}+\Gamma+\mathrm{i}$; parameters and proportion of invariant sites calculated by modelTest; four gamma rate categories, 20 replicates, $33 \mathrm{~h}$ time limit per replicate). Bootstrap support values for both datasets were estimated using 1000 replicates for a distance analysis using an ML model of substitution. Sequences have been deposited in GenBank under accession numbers AY620252-AY620366.
For reasons of space, not all sequences were included in the analyses shown. Seventy-nine out of a total of 91 named sequences were included. A total of 308 new partial environmental sequences were initially aligned with these; this number was reduced to 291 by eliminating chimaeras and sequences that could not be fully sequenced. This number was reduced further by removing effectively identical sequences; these were arbitrarily defined as those with fewer than 3-5 nucleotide differences in the most variable region of the $18 \mathrm{~S}$ rRNA gene (depending on their distribution between relatively more or less conserved regions), between approximate positions 625 and 740 in this alignment. As the fragments were mostly single-strand sequences, this allowed for PCR and sequencing errors. A final total of 110 environmental sequences was reached by cutting out many of those that grouped robustly within sufficiently well-represented clades.

\section{RESULTS AND DISCUSSION}

\section{Cercozoan diversity}

The provenances of samples used to construct the libraries represented by the alphanumeric codes on the phylogenetic trees (see Figs 2 and 3) are given in Table 1. The relationships between the total number of clones sequenced and the total numbers of clearly different sequences obtained from successive libraries is given in Table 2. These data are visualized in the species accumulation graphs (Fig. 1a-c).

Table 2. Sequence retrieval from all 41 environmental libraries

Refer to Table 1 for explanation of library codes.

\begin{tabular}{|c|c|c|c|c|c|}
\hline $\begin{array}{l}\text { Library } \\
\operatorname{code}(s)\end{array}$ & $\begin{array}{l}\text { Sequences } \\
\qquad(n)\end{array}$ & $\begin{array}{c}\text { Cumulative } \\
\text { sequences }(n)\end{array}$ & $\begin{array}{l}\text { Distinct lineages } \\
\text { in library }(n)\end{array}$ & $\begin{array}{l}\text { Novel lineages } \\
\text { in library }(n)\end{array}$ & $\begin{array}{c}\text { Cumulative novel } \\
\text { lineages }(n)\end{array}$ \\
\hline PP1 & 20 & 20 & 13 & 13 & 13 \\
\hline PP2 & 5 & 25 & 4 & 2 & 15 \\
\hline $\mathrm{C}$ & 17 & 42 & 13 & 13 & 28 \\
\hline $12-3,-4$ & 11 & 53 & 9 & 7 & 35 \\
\hline B & 12 & 65 & 6 & 6 & 41 \\
\hline $12-1,-2$ & 11 & 76 & 5 & 4 & 45 \\
\hline $7-1,-2$ & 10 & 86 & 10 & 10 & 55 \\
\hline $7-3,-4$ & 12 & 98 & 9 & 7 & 62 \\
\hline $7-5,-6$ & 11 & 109 & 11 & 10 & 72 \\
\hline A & 21 & 130 & 12 & 9 & 81 \\
\hline $\mathrm{D}$ & 10 & 140 & 9 & 7 & 88 \\
\hline $\mathrm{F}$ & 8 & 148 & 6 & 6 & 94 \\
\hline $\mathrm{E}$ & 12 & 160 & 2 & 2 & 96 \\
\hline $13-3,-4$ & 10 & 170 & 7 & 7 & 103 \\
\hline $9-5,-6$ & 6 & 176 & 5 & 5 & 108 \\
\hline $13-1,-2$ & 11 & 187 & 9 & 8 & 116 \\
\hline 8 & 18 & 205 & 9 & 7 & 123 \\
\hline $10-3$ & 7 & 212 & 5 & 3 & 126 \\
\hline $4-5,-6$ & 15 & 227 & 10 & 8 & 134 \\
\hline $10-4$ & 6 & 233 & 3 & 0 & 134 \\
\hline $9-3,-4$ & 13 & 246 & 7 & 6 & 140 \\
\hline $9-1,-2$ & 14 & 260 & 9 & 8 & 148 \\
\hline $4-1,-2$ & 14 & 274 & 10 & 8 & 156 \\
\hline $4-3,-4$ & 9 & 283 & 9 & 9 & 165 \\
\hline 6 & 8 & 291 & 7 & 3 & 168 \\
\hline
\end{tabular}



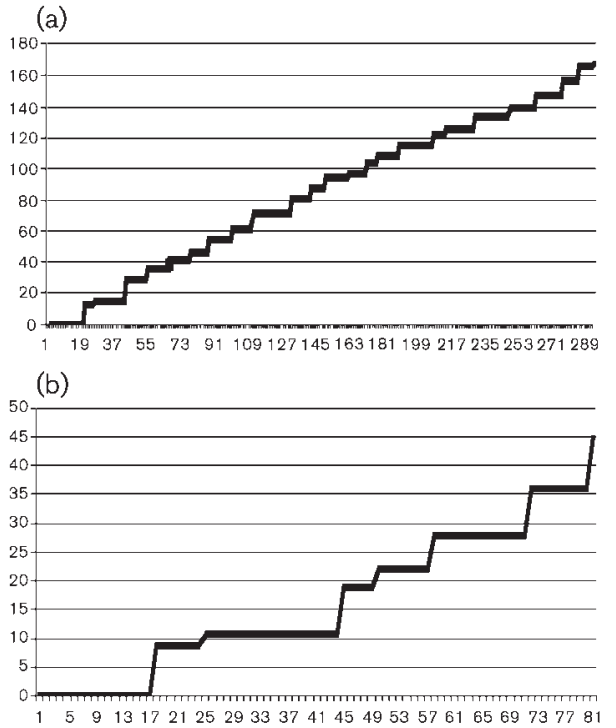

(c)

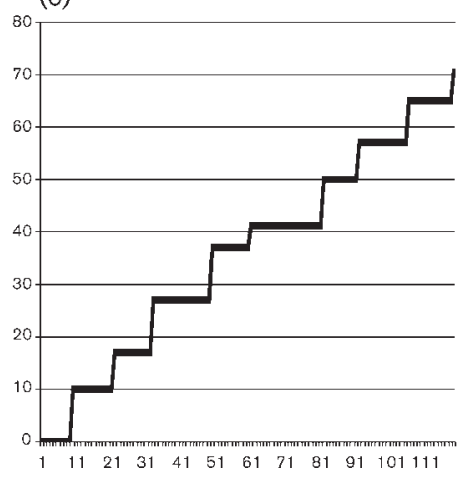

Fig. 1. Species accumulation graphs: (a) across all libraries, (b) from Priest Pot libraries only; (c) from marine libraries only. The $x$-axes show the number of sequences sampled and the $y$-axes show the number of novel lineages produced by each successive library. Refer to Table 1 for details of individual libraries.

Such is the diversity remaining unsampled, even within habitats (Tables 3 and 4; Fig. 1b, c), that none of these graphs shows any measurable sign of levelling off, and the gradients within habitats $(1 \cdot 80$ for Priest Pot libraries; 1.68 for marine libraries) are very similar to that across all habitats $(1 \cdot 73)$. It would, therefore, be unsound to attempt an extrapolation to estimate the total number of lineages in the phylum. Within libraries, the mean incidence of duplicate hits $(31.6 \%)$ is more than twice that between libraries after within-library duplicates have been removed $(15 \cdot 6 \%)$. This difference is probably due to two factors: diversity per sample being restricted by the relatively small amount of material taken, and ligation and selection of multiple amplicons of the same initial molecule. Also, a high frequency of multiple hits within a library is expected, even when small numbers of clones are picked, if some genotypes are much more abundant or easily amplified than others. The relative contribution of these factors is unknown. But, if the most frequently amplified sequences within libraries differ from sample to sample, as they appear to, our strategy of sequencing relatively small numbers of sequences per library $($ mean $=11 \cdot 64)$ from a large number of libraries (41) is likely to reveal more of the real diversity both at single sites and between sites than one of sequencing many clones from a small number of libraries. The mean incidence of between-library duplicate hits within habitats (Tables 3 and 4; Fig. 1b, c) is $20 \%$, higher than the $15.6 \%$ across all libraries, suggesting that some taxa may exhibit habitat specificity or preferences. Although this difference is slight, it is unlikely to be due to the temporal aspect of sample collection, as both Priest Pot and marine samples were taken at many different times of the year.

Even so, our sampling is not fully random. With only one exception, two or three libraries were constructed from samples taken at the same site, and the choice of sites overall represents a relatively restricted set of ecological conditions (Table 1). If the selection of sampling sites was truly random, we would expect an even higher rate of detecting novel sequences than the $57 \cdot 7 \%$ here. Our approach will also underestimate true environmental diversity if sequence retrieval is biased towards those that are most abundant at the time of sampling as well as those most easily amplified by PCR. If the combined effect of these two factors is multiplicative, then the proportion of

Table 3. Sequence retrieval from libraries constructed from all Priest Pot samples

\begin{tabular}{|lccccc|}
\hline $\begin{array}{l}\text { Library } \\
\text { code }(\mathbf{s})\end{array}$ & $\begin{array}{c}\text { Sequences } \\
(\boldsymbol{n})\end{array}$ & $\begin{array}{c}\text { Cumulative } \\
\text { sequences }(\boldsymbol{n})\end{array}$ & $\begin{array}{c}\text { Distinct lineages } \\
\text { in library }(\boldsymbol{n})\end{array}$ & $\begin{array}{c}\text { Novel lineages } \\
\text { in library }(\boldsymbol{n})\end{array}$ & $\begin{array}{c}\text { Cumulative novel } \\
\text { lineages }(\boldsymbol{n})\end{array}$ \\
\hline 8 & 18 & 18 & 9 & 9 & 9 \\
$10-3$ & 7 & 25 & 5 & 2 & 11 \\
PP1 & 20 & 45 & 13 & 8 & 19 \\
PP2 & 5 & 50 & 4 & 3 & 22 \\
F & 8 & 58 & 6 & 6 & 28 \\
$4-1,-2$ & 14 & 72 & 10 & 8 & 45 \\
$4-3,-4$ & 9 & 81 & 9 & 9 & \\
\hline
\end{tabular}


Table 4. Sequence retrieval from libraries constructed from all marine samples

\begin{tabular}{|lccccc|}
\hline $\begin{array}{l}\text { Library } \\
\text { code(s) }\end{array}$ & $\begin{array}{c}\text { Sequences } \\
(\boldsymbol{n})\end{array}$ & $\begin{array}{c}\text { Cumulative } \\
\text { sequences }(\boldsymbol{n})\end{array}$ & $\begin{array}{c}\text { Distinct lineages } \\
\text { in library }(\boldsymbol{n})\end{array}$ & $\begin{array}{c}\text { Novel lineages } \\
\text { in library }(\boldsymbol{n})\end{array}$ & $\begin{array}{c}\text { Cumulative novel } \\
\text { lineages }(\boldsymbol{n})\end{array}$ \\
\hline $7-1,-2$ & 10 & 10 & 10 & 10 & 10 \\
$7-3,-4$ & 12 & 22 & 9 & 7 & 17 \\
$7-5,-6$ & 11 & 33 & 11 & 10 & 27 \\
C & 17 & 50 & 13 & 10 & 37 \\
$12-3,-4$ & 11 & 61 & 9 & 4 & 41 \\
A & 21 & 82 & 12 & 9 & 50 \\
D & 10 & 92 & 9 & 7 & 57 \\
$9-1,-2$ & 14 & 106 & 9 & 8 & 65 \\
$09-3,-4$ & 13 & 119 & 7 & 6 & 71 \\
\hline
\end{tabular}

diversity detectable without uneconomically large amounts of colony screening may be surprisingly low.

Of the 79 named sequences on Fig. 2, at least 62 are clearly different from each other. Of our 168 distinct environmental sequences, only one is putatively identical to a named sequence. Considering (i) the shapes and gradients of the species accumulation curves, (ii) the likelihood of restricted and biased detection inherent in our approach, as described above, (iii) that the distinction between genetically discrete entities is being made at the relatively crude level of $18 \mathrm{~S}$ rRNA and (iv) the fact that only $1 / 62$ $(1 \cdot 6 \%)$ of named sequences were matched by any of the 168 distinct environmental sequences, we suggest that our demonstrated total of approximately 230 distinct cercozoan genetic lineages is one or two orders of magnitude smaller than the real total. If the environmental and named lineages could be considered independent random samples, the total diversity would exceed 10000 lineages, but they are clearly not and we have insufficient data to attempt a reliable estimate.

Further evidence for gross undersampling in this study is provided by unpublished results with primers specific to clades within the phylum, showing that similar levels of hidden diversity are also found at lower levels of the taxonomic hierarchy (D. Bass, unpublished). An interesting comparison can be made with ciliates, with approximately 3000 free-living morphospecies (Finlay et al., 1996). However, if all ciliate morphospecies contain as many sibling species (probably representing ecologically differentiated evolutionary units) as, for example, Paramecium and Tetrahymena, the total number of ciliate 'species' should be at least an order of magnitude higher (Nanney, 1999). Similarly, in the protozoan phylum with the largest number of described morphospecies, Foraminifera (Corliss, 1999), there is also evidence for cryptic genetic diversity (Pawlowski et al., 2001; Holzmann et al., 2003). We cannot make comparisons between sibling species in ciliates and lineages in Cercozoa, as we know almost nothing about the sexuality of any free-living cercozoan, but we suggest that the fact that Ciliophora and Foraminifera have more described species than Cercozoa may be a function of the intensity with which they have been studied, rather than being a reflection of real levels of biodiversity among these phyla. Our phylum-specific sampling suggests that broader, often eukaryote-wide, environmental surveys (e.g. Moonvan der Staay et al., 2001; López-García et al., 2001, 2003; Edgcomb et al., 2002; Díez et al., 2001; Dawson \& Pace, 2002; Amaral Zettler et al., 2002; Stoeck \& Epstein, 2003) are more undersampled than previously suspected, even allowing for the fact that they probably underestimate diversity in many branches of the eukaryote tree owing to experimental biases.

\section{Novel clades}

Our environmental libraries have revealed at least nine novel cercozoan clades (NC; i.e. currently containing no cultured representatives; indicated on Figs 2 and 3), some of which may be shown by further work to merit the status of order or above. However, such designation will not be possible until members of each novel clade are brought into culture and characterized morphologically. The mostly low bootstrap support values indicate that their correct positions may be elsewhere on the tree, perhaps within other, characterized, clades in some cases. Conversely, some of the uncharacterized clades shown in Fig. 2 to be nested within established groups (not marked) may prove not to belong there and in fact be novel clades in their own right. Other analyses (not shown) illustrate this: 9-2.2 (NC4 on Fig. 2) can group within NC3, the rest of NC4 (7-6.2 and 9-1.4) can group with moderate support within Proteomyxidea and D6 and 13-2.8, a clade within Basal 'Group' T, can become an outgroup to Metopiida and NC6 with $23 \%$ support. Furthermore, the novel clades that are sisters to single known groups might be shown actually to belong to those groups. These uncertainties will probably not be resolved until the organisms they represent are isolated and other (e.g. protein-coding) genes are used to improve the phylogenetic analysis.

As noted above, several recent studies have shown that many previously unclassified protozoa are in fact cercozoans. Probably many more await discovery and description. Many, if not most, heterotrophic zooflagellates of unknown affinity may prove to belong to the phylum, 


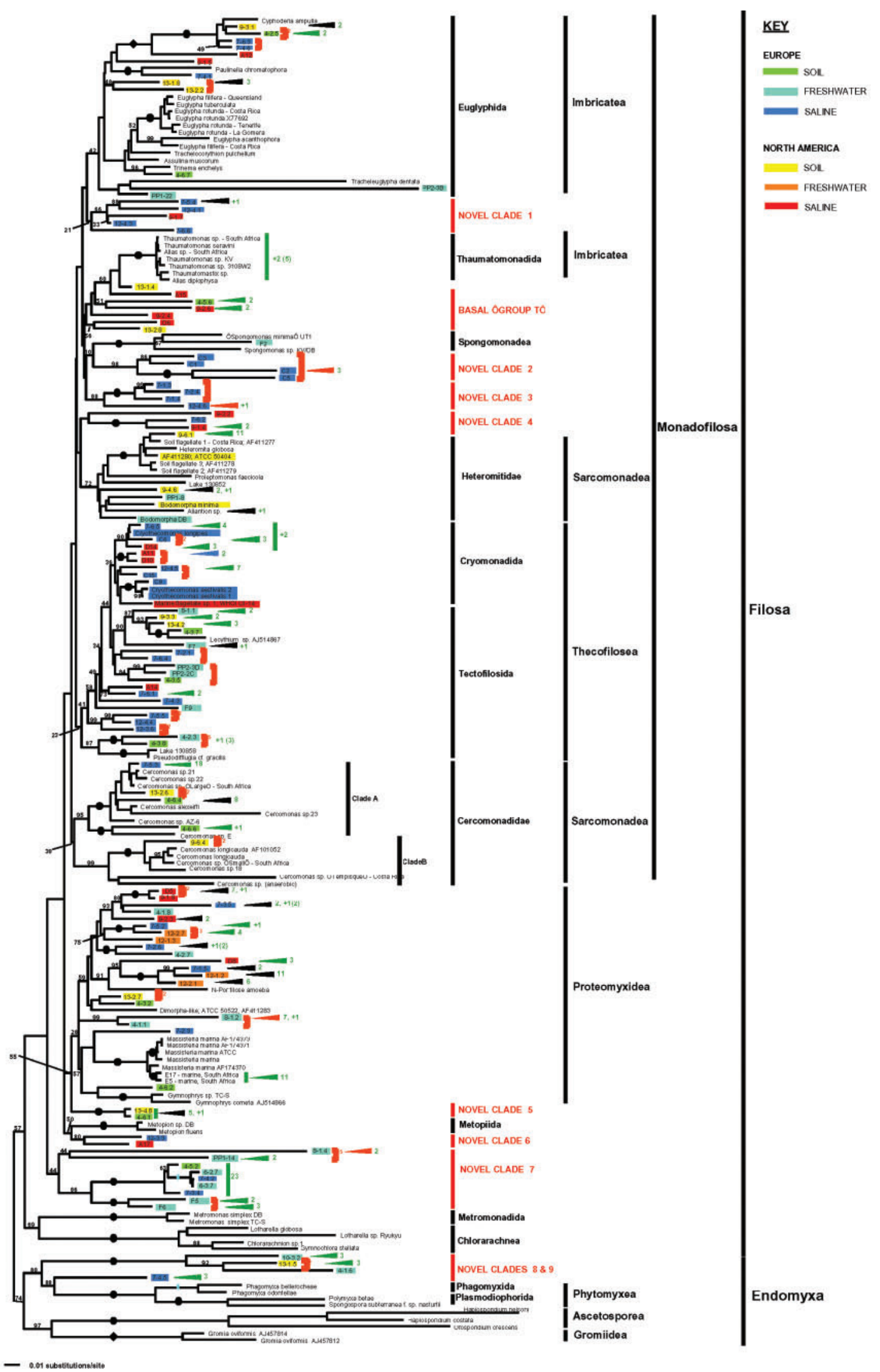


Fig. 2. Distance tree of 189 cercozoan $18 \mathrm{~S}$ rRNA gene sequences (110 of which are from environmental libraries) using 1164 positions (minimum evolution; GTR $\Gamma+\mathrm{i}$ model: $\alpha=0.666589 ; \mathrm{i}=0.200474$ ). Orange brackets refer to clades of distinct sequences recovered exclusively from the same library or sampling sites. Where only one member of such clades is shown on the tree (i.e. the bracket only contains one taxon), the total number in the clade is given as a subscript to the bracket. Refer to Methods for the rationale for selection of sequences shown in this tree. Numbers in green at the end of green wedges give the number of identical or effectively indistinguishable sequences corresponding to the one marked. Green numbers preceded by ' + ' indicate other distinct lineages in the clade indicated that were omitted from the analysis for practical reasons. For example, '+1 (3)' indicates that there are three indistinguishable copies of one additional distinct sequence in the clade marked. Sequences from pure cultures or databases are indicated by name and/or reference number, those from environmental libraries by codes, for details of which refer to Table 1. Coloured boxes describe the provenance of the environmental sequences, as described by the key. All bootstrap percentages $>84 \%$ are shown. In addition, for the novel clades, bootstrap values are given for the basal nodes and for all values $>50 \%$ within the clades, where possible. Black dots indicate $100 \%$ support.

including those that exhibit both flagellate and other, more sessile forms (for example Dimorpha), as well as most filose testate amoebae, many naked filose and reticulate amoebae (excluding, for example, the filose opisthokont nucleariid amoebae) and many forms with radially symmetrical arrangements of filopods, with and without mineralized tests and axopodia-like structures (e.g. Desmothoracida). Even since this paper was submitted, we have found that Pseudopirsonia, a very recently described marine zooflagellate (Kühn et al., 2004), is nested firmly within our marine NC1. Thus, Pseudopirsonia is not a heterokont as stated in GenBank, but yet another cercozoan. This also confirms that NC1 did represent a novel group of cercozoans. However, sequences of Phaeodarea, which also became available after this paper was completed and have turned out to be Cercozoa (Polet et al., 2004), are clearly deepbranching Filosa but not part of any novel clade; they branch near the base of Tectofilosida, grouping in a preliminary analysis with the clade of four sequences including Pseudodifflugia.

\section{Ecology and distribution}

We constructed libraries from samples taken from (i) different ecological conditions and (ii) similar habitats on different continents, in order to ascertain whether sequences clustered together according to ecological and/or geographical provenance, to the exclusion of all other sequences known. Interpretation of the resulting patterns of clustering is made difficult by the degree of undersampling of the phylum as a whole, but several points are striking. Some clades comprise sequences from only one habitat and/or one sampling site. In the latter case, such sequences may either be from the same library or from multiple libraries taken from the same locality. Examples of these are shown on Fig. 2 by square orange brackets. Where sequences not shown on Fig. 2 group exclusively with other, different, sequences from the same locality, the number of sequences involved is given by an orange subscript number to the bracket. There are at least 22 instances of this from the 41 libraries constructed - an unexpectedly large number if a very large number of taxa are randomly distributed around the globe (Finlay, 2002).
A possible initial inference of this was that these clusters represent geographically restricted taxa; i.e. some barrier to dispersal is restricting gene flow (dispersal), leading to local radiations of lineages. This can be tested by designing PCR primers specific to the clades in question and screening samples from a range of habitats from around the world. We did this for NC3 and the clade represented by F5 and F6 in NC7, with environmental DNA extractions from five biogeographical regions (Nearctic, Neotropical, Oceanian, Palaearctic, Ethiopian). Bands representing NC3 [which was initially found only in UK (Portsmouth) marine samples; see Fig. 2] were produced from approximately $90 \%$ of environmental total DNA extractions from both marine and non-marine environments in all biogeographical regions. The F5/F6 clade only produced bands for about $4 \%$ of DNA samples, indicating that its distribution is much more patchy, but was also amplified from both types of environment (D. Bass, unpublished results). Clearly, the inability of the initial screening with Cercozoaspecific primers to find sequences from these two clades in North American samples was the result of undersampling. To test this further, we made libraries from NC3 and the F5/F6 subset of NC7 from New Zealand and Panama. From the NC3 library, we retrieved sequences identical to the Portsmouth 7-1.4, and, from the NC7 subset library, sequences from New Zealand and Panama were identical to each other, but different from those originally obtained from Priest Pot. In addition, we have sequenced identical 18S rRNA genes from cultured Cercomonas strains isolated from Oxford and Panama (unpublished). Although this does not prove that all $18 \mathrm{~S}$ rRNA sequences are ubiquitous, it does show that some are identical on different continents. Therefore, we can reasonably infer that most, if not all, of the apparent geographical clustering suggested by Fig. 2 is the result of undersampling. Our results therefore do not support the hypothesis that these organisms show biogeographical structuring due to distance or physical barriers at the level of phylogenetic resolution provided by the $18 \mathrm{~S}$ rRNA gene. This experiment also shows that ecological preferences inferred from phylogenies of environmentally cloned sequences need thorough and intensive testing to eliminate artefactual patterns due to taxon undersampling. Further work could reveal whether lineages within the 


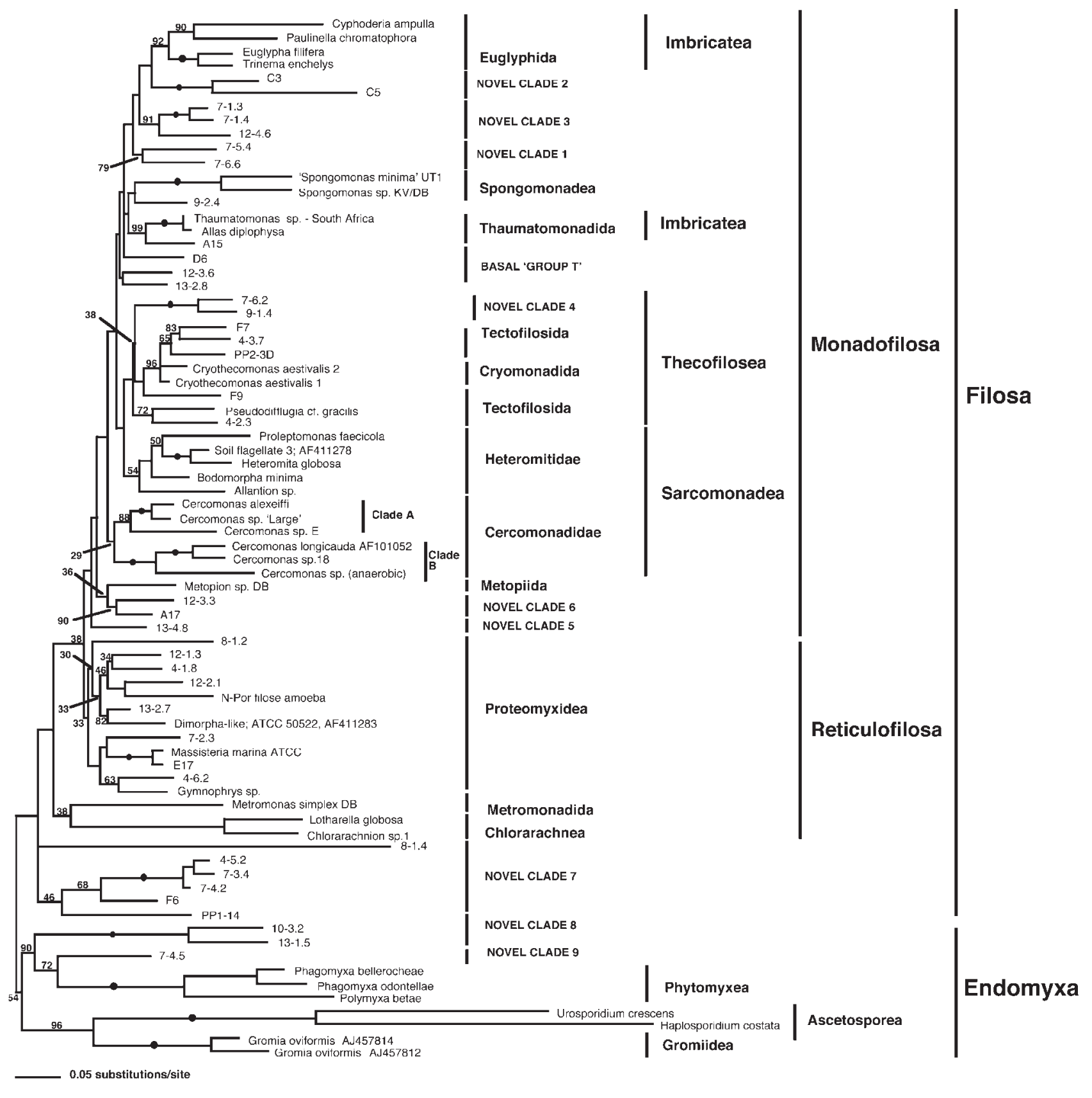

Fig. 3. $M L$ tree of 76 cercozoan 18S rRNA gene sequences using 1164 positions $(\Gamma+\mathrm{i}$ model: $\alpha=0 \cdot 503610$; $i=0.202384)$. The bootstrap percentages are support values for the distance tree with corresponding $\Gamma+i$ values analysed using an ML model of substitution. All support values $\geqslant 29 \%$ are shown (black dots indicate $100 \%$ support).

groups detected by these primers are restricted to one or other environment (and whether lineages differing only at the level of the ITS region of the rRNA gene, for example, show ecological segregation). A quantitative PCR approach could also be taken to see whether certain lineages occur preferentially in either environment. Other groups, for example Thecofilosea, would repay similar investigation: so far Cryomonadida contains only sequences from marine samples, whereas the paraphyletic 'basal Thecofilosea'
(Tectofilosida) contains those from all environments sampled, a preliminary result that is borne out by light microscope observations of cultures taken directly from the field.

However, the higher than expected incidence of sequences from a particular site clustering together deserves further investigation. It is known that the local: global species ratio for protozoa is high (Finlay, 2002) and that, by treating a 
single, small sample in a variety of ways, a much larger diversity of protozoa can be recovered than that revealed by observation of the initial crude sample (e.g. Esteban \& Finlay, 2003; Persson, 2002). In addition to these established patterns, a recent study has shown that, even within samples of soil as small as $8 \mathrm{~mm}^{3}$, there can be a clear structure to the distribution of different species of amoebae (Smirnov \& Thar, 2003). Forces that may shape spatial distributions of protozoa on small scales have been little studied and may include, in the light of the extraordinary richness of local diversity, functionally defined consortia of related taxa that always occur together and ecological optimization into quite specific realized niches even where the individual taxa involved have relatively broad ecological tolerances. There is also a temporal aspect to the heterogeneous distribution of lineages; different sets of taxa are found at the same site at different times of the year (Bojanic, 2001; Chen et al., 2002) and at different stages of succession in colonizing a habitat (Medlin et al., 1985), so it is possible that sets of taxa occur simultaneously that are more closely related to each other (and show common adaptations) than to those that occur at other times of the year. This would be the expected result of competitive speciation within lineages of organisms occurring simultaneously in the same stable environment type over long periods of time, where the advantages of being active at that particular time outweigh other competitive disadvantages that could be minimized by evolutionary displacement.

\section{Cercozoan phylogeny}

Four new sequences from isolated strains of three genera are presented in this paper.

(i) The sequences of Metromonas simplex DB and Metromonas simplex TC-S are very similar to each other, although not identical, and were obtained from morphologically similar strains. Although the sister relationship of these sequences to Chlorarachnea is moderately well supported by the distance tree (Fig. 2), it is only weakly supported by the ML tree (Fig. 3), and Metromonas shares no obvious structural characters with this group. Because of this, plus a lack of structural affinity with proteomyxids and the propensity of Metromonas to glide on its posterior flagellum in motile form (shared with thaumatomonads and sarcomonads), we place the genus in the new order Metromonadida, within Monadofilosa (see below); the grouping of the Metromonas sequences with the longbranch chlorarachneans might be artefactual, as they also have longer branches than many Monadofilosa.

(ii) We have sequenced the $18 \mathrm{~S}$ rRNA gene from a new isolate of Spongomonas (Spongomonas sp. KV/DB), which groups strongly with Spongomonas minima UT1. It was previously unclear whether Spongomonas minima UT1 was actually a Spongomonas because its flagella do not resemble those of other Spongomonas isolates under the light microscope (Cavalier-Smith \& Chao, 2003), although its general cell structure is similar to that of Spongomonas (CavalierSmith et al., 2004). The strong grouping of these two taxa now establishes Spongomonadea in Monadofilosa. Due to a labelling error of two DNA samples, Metromonas simplex TC-S was mistakenly designated Spongomonas sp. 7A in Cavalier-Smith (2000) and Cavalier-Smith \& Chao (2003). Because of this, Spongomonadida was wrongly placed in Reticulofilosa (Cavalier-Smith, 2000). On Figs 2 and 3, Spongomonadida appears to be weakly related to Thaumatomonadida. However, this part of the tree is rather unstable and bootstrap supports are weak. It is possible that Spongomonadida is more closely related to Cryomonadida, as both have two double ciliary transition region plates. This relationship is sometimes also supported by phylogenetic trees (Cavalier-Smith \& Chao, 2003).

The four marine-derived sequences $(\mathrm{C} 1, \mathrm{C} 2, \mathrm{C} 3$ and $\mathrm{C} 5)$ comprising a sister clade to the three Spongomonas sequences on Fig. 2 have no cultured relatives, and we cannot assume that they belong to Spongomonadea, especially in the light of their marine provenance, from which environment Spongomonas has never yet been isolated. Therefore, this group is designated NC2. Its position on the tree is uncertain; there is no bootstrap support for its being sister to Spongomonadea and, in the ML tree (Fig. 3), its position as sister clade to the imbricate order Euglyphida is as poorly supported.

(iii) The more complete $18 \mathrm{~S}$ rRNA gene sequence for Metopion (covering the full region of the alignment used in this study) shows it to be a very divergent filosan. We suggest that Metopiida should be removed from Sarcomonadea (Cavalier-Smith \& Chao, 2003) and remain incertae sedis within Filosa until more data are available. Sequences 12-3.3 and A17 form a moderately supported sister clade (NC6) to Metopiida within Monadofilosa and, in some analyses (not shown), D6 and 13-2.8 (part of Basal 'Group' $\mathrm{T}$ in Fig. 2) are an outgroup to these four taxa, but with little support.

Once members of the novel, uncharacterized, clades are cultured, we can test the robustness and monophyly of the current higher taxa within Cercozoa. For example, if the uncultured putative members of Tectofilosida have an organic flexible tectum or rigid test with one or two apertures for filopodia, with or without agglutinated foreign particles, as do Pseudodifflugia and Lecythium, then the diagnosis for Thecofilosea would stand and would encompass this entire clade. Morphological data may help to clarify the position of NC4 more easily and definitively than further phylogenetic analysis; the ML tree (Fig. 3) and some distance trees (not shown) place NC4 in Tectofilosida with $38 \%$ support, whereas the distance tree (Fig. 2) shows it as sister (with negligible support) to Heteromitidae. Similarly, are NC1, 2 and 3 true imbricates? The likelihood of this is weakened by the presence of Spongomonadea within the imbricate clade; Spongomonas shows no specific morphological affinity with the imbricates. 
Novel clades that are also basal branches in the cercozoan tree would particularly repay further investigation. NC7 appears to be relatively common in a range of environments, so may be relatively easy to isolate; genetic and morphological analysis may clarify the relationships of Chlorarachnea, Metromonadida and Metopiida to the rest of Filosa. In the light of the relatively strong support for a sister relationship between NCs 8 and 9 and Phytomyxea, further work should be undertaken to determine whether these novel taxa are parasitic or endosymbiotic. This is important for understanding of the evolution of symbiosis within Cercozoa and might reveal economically significant species associations.

\section{PCR chimaera formation}

It is well known that recombination between partially homologous templates can occur during PCR, by templateswitching during a single PCR cycle or by truncated extension products annealing to partially homologous templates in subsequent cycles (Odelberg et al., 1995). This has been shown to be particularly prevalent in polyploids, due to the presence of partial homologues (Cronn et al., 2002). Modifications to PCR cycling conditions known to reduce the proportion of chimaeras include long elongation times and as few cycles as possible, ideally to the mid-point of amplification (Judo et al., 1998). For all environmental $18 \mathrm{~S}$ rRNA libraries in this study, we used 3.5 min extensions for $1.2 \mathrm{~kb}$ and 30 cycles, which was found to be the minimum number required for reliable production of visible PCR products by gel electrophoresis across a range of diluted environmental DNA extractions.

To test empirically the rate of chimaera formation under these PCR conditions, we created an 18S rRNA gene library by amplifying a mixture of known cercozoan monoculture genomic DNAs, using the same primer pair as for the environmental libraries. Genomic DNAs used were Cercomonas longicauda, Cercomonas sp. AZ6, Bodomorpha sp., Heteromita sp., Allantion sp., Thaumatomonas sp., marine flagellate sp. 1; WHOI LII-14, Massisteria sp., Dimorpha-like and Chlorarachnion sp. (see Fig. 2). Out of $361 \cdot 2 \mathrm{~kb}$ sequences, two were definitely chimaeric $(5 \cdot 56 \%)$; the others appeared not to be. The two chimaeras each had one recombination site, respectively between positions of the fragment amplified 270-295 (left-hand end = Cercomonas longicauda; right-hand end = Cercomonas AZ-6) and 220-239 (left-hand end = Cercomonas AZ-6, right-hand end = Cercomonas longicauda). Interestingly, out of the ten genomic DNAs used, only five were represented in this library sample. Within those five, one taxon (Cercomonas longicauda) was represented 26 times, Cercomonas sp. AZ6 five times and Allantion, Heteromita and Thaumatomonas once each. This uneven representation of the ten genera may have several causes, between which we cannot distinguish: biased amplification of certain sequences, different levels of inhibitor or degradation in the monoculture genomic DNA samples and differences between the relative proportions of eukaryote and prokaryote DNA in each sample (only the total amounts of DNA from each culture were equalized in the mixture that was used for cloning). However, we know from experiments with pure culturederived DNA that the primer pair used for our environmental screening works well on all of these genera.

This experiment reinforces our arguments that PCR from environmental samples is likely to undersample true diversity. It also shows that the formation of PCR chimaeras in studies like this is a potential problem, as may also be biased amplification. In our study, obvious chimaeras were removed from the analysis, but a small minority of the remaining sequences might be undetected chimaeras, for example those between very closely related lineages or those with a site of recombination close to either end of the amplified fragment. However, such infrequent artefacts will not significantly affect the topology of the tree and do not significantly inflate the inferred levels of diversity. We can therefore safely conclude that cercozoan diversity is very large, and mostly unknown.

\section{Diagnosis of Metromonadida ord. nov. Bass \& Cavalier-Smith 2004}

Superclass Monadofilosa. Flagellates with two apical flagella, a long one clearly visible under light microscope and a second stub. Motile form glides smoothly with flagellum trailing behind; sessile/feeding form attached to substrate by posterior end of long flagellum, the proximal end of which oscillates the cell regularly like a metronome. At least two species, marine.

\section{Diagnosis of family Metromonadidae fam. nov. Bass \& Cavalier-Smith 2004}

Diagnosis as for the order. Etymology: Gr. n. metron measure; Gr. adj. monos single, alone; based on the type genus Metromonas. Type species Metromonas simplex (Griessmann) Larsen \& Patterson 1990.

\section{ACKNOWLEDGEMENTS}

We thank Ema Chao for providing many of the sequences already in our database, Professor Bland Finlay for assistance with sample collection and Professor Keith Vickerman for the culture of Spongomonas sp. KV/DB. T. C.-S. and D. B. designed the experiment, D. B. undertook the laboratory work and both authors contributed to the discussion. The work was supported by a NERC Research Studentship to D. B. T. C.-S. thanks NERC UK for research grants and the Canadian Institute for Advanced Research and NERC UK for fellowship support.

\section{REFERENCES}

Amaral Zettler, L. A., Gomez, F., Zettler, E., Keenan, B. G., Amils, R. \& Sogin, M. L. (2002). Microbiology: eukaryotic diversity in Spain's River of Fire. Nature 417, 137.

Archibald, J. M., Longet, D., Pawlowski, J. \& Keeling, P. J. (2003). A novel polyubiquitin structure in Cercozoa and Foraminifera: evidence for a new eukaryotic supergroup. Mol Biol Evol 20, 62-66. 
Atkins, M. S., Teske, A. P. \& Anderson, O. R. (2000). A survey of flagellate diversity at four deep-sea hydrothermal vents in the Eastern Pacific Ocean using structural and molecular approaches. J Eukaryot Microbiol 47, 400-411.

Bojanic, N. (2001). Seasonal distribution of the ciliated protozoa in Kastela Bay. J Mar Biol Assoc UK 81, 383-390.

Burki, F., Berney, C. \& Pawlowski, J. (2002). Phylogenetic position of Gromia oviformis Dujardin inferred from nuclear-encoded small subunit ribosomal DNA. Protist 153, 251-260.

Cavalier Smith, T. (1996/7). Amoeboflagellates and mitochondrial cristae in eukaryotic evolution: megasystematics of the new protozoan subkingdoms Eozoa and Neozoa. Arch Protistenk 147, 237-258.

Cavalier-Smith, T. (1998a). Neomonada and the origin of animals and fungi. In Evolutionary Relationships among Protozoa, pp. 375407. Edited by G. H. Coombs, K. Vickerman, M. A. Sleigh \& A. Warren. London: Chapman \& Hall.

Cavalier-Smith, T. (1998b). A revised six-kingdom system of life. Biol Rev 73, 203-266.

Cavalier-Smith, T. (2000). Flagellate megaevolution: the basis for eukaryote diversification. In The Flagellates, pp. 361-390. Edited by J. R. Green \& B. S. C. Leadbeater. London: Taylor \& Francis.

Cavalier-Smith, T. (2004). Only six kingdoms of life. Proc R Soc Lond B Biol Sci 271, 1251-1262.

Cavalier-Smith, T. \& Chao, E. E-Y. (2003). Phylogeny and classification of phylum Cercozoa (Protozoa). Protist 154, 341-358.

Cavalier-Smith, T., Chao, E. E-Y. \& Oates, B. (2004). Molecular phylogeny of Amoebozoa and the evolutionary significance of the unikont Phalansterium. Eur J Protistol 40, 21-48.

Chen, C., Ji, R., Schwab, D. J. \& 11 other authors (2002). A model study of the coupled biological and physical dynamics in Lake Michigan. Ecol Model 152, 145-268.

Corliss, J. O. (1999). Biodiversity and numbers of species of protists. In Nature and Human Society: the Quest for a Sustainable World pp. 130-155. Edited by P. Raven \& T. Williams. Washington, DC: National Academy Press.

Cronn, R., Cedroni, M., Haselkorn, T., Grover, C. \& Wendel, J. F. (2002). PCR-mediated recombination in amplification products derived from polyploid cotton. Theor Appl Genet 104, 482-489.

Dawson, S. C. \& Pace, N. R. (2002). Novel kingdom-level eukaryotic diversity in anoxic environments. Proc Natl Acad Sci U S A 99, 8324-8329.

Díez, B., Pedrós-Alió, C. \& Massana, R. (2001). Study of genetic diversity of eukaryotic picoplankton in different oceanic regions by small-subunit rRNA gene cloning and sequencing. Appl Environ Microbiol 67, 2932-2941.

Edgcomb, V. P., Kysela, D. T., Teske, A., de Vera Gomez, A. \& Sogin, M. L. (2002). Benthic eukaryotic diversity in the Guaymas Basin hydrothermal vent environment. Proc Natl Acad Sci U S A 99, 7658-7662.

Embley, T. M. \& Hirt, R. P. (1998). Early branching eukaryotes? Curr Opin Genet Dev 8, 624-629.

Esteban, G. F. \& Finlay, B. J. (2003). Cryptic freshwater ciliates in a hypersaline lagoon. Protist 154, 411-418.

Finlay, B. J. (2002). Global dispersal of free-living microbial eukaryote species. Science 296, 1061-1063.

Finlay, B. J., Corliss, J. O., Esteban, G. \& Fenchel, T. (1996). Biodiversity at the microbial level: the number of free-living ciliates in the biosphere. Q Rev Biol 71, 221-237.

Holzmann, M., Habura, A., Giles, H., Bowser, S. S. \& Pawlowski, J. (2003). Freshwater foraminiferans revealed by analysis of environmental DNA samples. J Eukaryot Microbiol 50, 135-139.
Horner, D. S. \& Embley, T. M. (2001). Chaperonin 60 phylogeny provides further evidence for secondary loss of mitochondria among putative early-branching eukaryotes. Mol Biol Evol 18, 1970-1975.

Judo, M. S. B., Wedel, A. B. \& Wilson, C. (1998). Stimulation and suppression of PCR-mediated recombination. Nucleic Acids Res 26, 1819-1825.

Keeling, P. J. (2001). Foraminifera and Cercozoa are related in actin phylogeny: two orphans find a home? Mol Biol Evol 18, 1551-1557.

Kühn, S., Lange, M. \& Medlin, L. K. (2000). Phylogenetic position of Cryothecomonas inferred from nuclear-encoded small subunit ribosomal RNA. Protist 151, 337-345.

Kühn, S., Medlin, L. \& Eller, G. (2004). Phylogenetic position of the parasitoid nanoflagellate Pirsonia inferred from nuclear-encoded small subunit ribosomal DNA and a description of Pseudopirsonia n. gen. and Pseudopirsonia mucosa (Drebes) comb. nov. Protist 155, 143-156.

Larsen, J. \& Patterson, D. J. (1990). Some flagellates (Protista) from tropical marine sediments. J Nat Hist 24, 801-937.

Longet, D., Archibald, J. M., Keeling, P. J. \& Pawlowski, J. (2003). Foraminifera and Cercozoa share a common origin according to RNA polymerase II phylogenies. Int J Syst Evol Microbiol 53, 1735-1739.

López-García, P., Rodríguez-Valera, F., Pedrós-Alió, C. \& Moreira, D. (2001). Unexpected diversity of small eukaryotes in deep-sea Antarctic plankton. Nature 409, 603-607.

López-García, P., Philippe, H., Gail, F. \& Moreira, D. (2003). Autochthonous eukaryotic diversity in hydrothermal sediment and experimental microcolonizers at the Mid-Atlantic Ridge. Proc Natl Acad Sci U S A 100, 697-702.

Martin, D. \& Rybicki, E. (2000). RDP: detection of recombination amongst aligned sequences. Bioinformatics 16, 562-563.

Medlin, L. K., Fryxell, G. A. \& Cox, E. R. (1985). Successional sequences of microbial colonization on three species of rhodophycean macroalgae. Ann Bot Lond 56, 399-414.

Moon-van der Staay, S. Y., De Wachter, R. \& Vaulot, D. (2001). Oceanic $18 \mathrm{~S}$ rDNA sequences from picoplankton reveal unsuspected eukaryotic diversity. Nature 409, 607-610.

Moreira, D. \& López-García, P. (2002). The molecular ecology of microbial eukaryotes unveils a hidden world. Trends Microbiol 10, 31-38.

Nanney, D. L. (1999). When is a rose? The kinds of tetrahymenas. In Species: New Interdisciplinary Essays. Edited by R. W. Wilson. Cambridge, MA: MIT Press.

Nikolaev, S. I., Berney, C., Fahrni, J., Mylnikov, A. P., Aleshin, V. V., Petrov, N. B. \& Pawlowski, J. (2003). Gymnophrys cometa and Lecythium sp. are core Cercozoa: evolutionary implications. Acta Protozool 42, 183-190.

Odelberg, S. J., Weiss, R. B., Hata, A. \& White, R. (1995). Templateswitching during DNA synthesis by Thermus aquaticus DNA polymerase I. Nucleic Acids Res 23, 2049-2057.

Pawlowski, J., Fahrni, J. F., Brykczynska, U., Habura, A. \& Bowser, S. S. (2001). Molecular data reveal high taxonomic diversity of allogromiid Foraminifera in Explorers Cove (McMurdo Sound, Antarctica). Polar Biol 25, 96-105.

Persson, A. (2002). Proliferation of cryptic protists and germination of resting stages from untreated sediment samples with emphasis on dinoflagellates. Ophelia 55, 151-166.

Philippe, H. \& Germot, A. (2000). Phylogeny of eukaryotes based on ribosomal RNA: long-branch attraction and models of sequence evolution. Mol Biol Evol 17, 830-834. 
Polet, S., Berney, C., Fahrni, J. \& Pawlowski, J. (2004). Smallsubunit ribosomal RNA gene sequences of Phaeodarea challenge the monophyly of Haeckel's Radiolaria. Protist 155, 53-63.

Posada, D. \& Crandall, K. A. (1998). MODELTEST: testing the model of DNA substitution. Bioinformatics 14, 817-818.

Richards, T., Hirt, R. P., Williams, A. P. \& Embley, T. M. (2003). Horizontal gene transfer and the evolution of parasitic protozoa. Protist 154, 17-32.

Smirnov, A. \& Thar, R. (2003). Spatial distribution of Gymnamoebae (Rhizopoda, Lobosea) in brackish-water sediments at the scale of centimeters and millimeters. Protist 154, 359-369.

Stiller, J. W. \& Hall, B. D. (1999). Long-branch attraction and the rDNA model of early eukaryotic evolution. Mol Biol Evol 16, 1270-1279.
Stoeck, T. \& Epstein, S. (2003). Novel eukaryotic lineages inferred from small-subunit rRNA analyses of oxygen-depleted marine environments. Appl Environ Microbiol 69, 2657-2663.

Swofford, D. W. (1999). PAUP* 4.0b10. Sunderland, MA: Sinauer.

Vickerman, K., Le Ray, D., Hoef-Emden, K. \& De Jonckheere, J. (2002). The soil flagellate Proleptomonas faecicola: cell organisation and phylogeny suggest that the only described free-living trypanosomatid is not a kinetoplastid but has cercomonad affinities. Protist 153, 9-24.

Wylezich, C., Meisterfeld, R., Meisterfeld, S. \& Schlegel, M. (2002). Phylogenetic analyses of small subunit ribosomal RNA coding regions reveal a monophyletic lineage of euglyphid testate amoebae (Order Euglyphida). J Eukaryot Microbiol 49, 108-118. 